\title{
Indole Alkaloids Synthesis via a Selective Cyclization of Aminocyclopropanes
}

\author{
Filippo De Simone§ and Jérôme Waser* \\ §SCS-DSM Award for best poster presentation
}

\begin{abstract}
The continuous progress in medicinal chemistry requires more versatile synthetic strategies for the generation of large libraries of active compounds and their analogues. As a result, the research for new effective cyclization and cycloaddition reactions is an essential task in organic chemistry. In 2008 we developed the first catalytic formal homo-Nazarov reaction starting from activated cyclopropanes. Herein we report the extension of the catalytic formal homo-Nazarov cyclization to aminocyclopropanes. Highly diastereoselective cyclizations were obtained via an acyliminium intermediate generated through opening of the cyclopropane. An excellent control over the regioselectivity of either the $\mathrm{C}-\mathrm{C}$ or $\mathrm{C}-\mathrm{N}$ cyclization in the case of free indoles as nucleophilic partners was achieved. The utility of the developed methodology was demonstrated by the generation of the polycyclic scaffolds of Aspidosperma and Gonioma natural products starting from a common intermediate. Based on this method, a formal total synthesis of the alkaloid aspidospermidine and the total synthesis of the alkaloid goniomitine are presented. Finally, the scope and limitations of our methodology are discussed on an extended range of vinyl-cyclopropyl ketones with cyclic or acyclic carbamates, as well as ethers as donor groups on the cyclopropane.
\end{abstract}

Keywords: Activated cyclopropanes · Alkaloids · Heterocycles $\cdot$ Regioselectivity $\cdot$ Total synthesis

\section{Introduction}

Carbocyclic and heterocyclic scaffolds play a key role in natural and pharmaceutical compounds. ${ }^{[1]}$ Rigidified ring systems assure a better stability and a well-defined tridimensional arrangement of functional groups allowing stronger and more selective interactions with receptors or enzymes. Consequently, the development of new efficient processes for the synthesis of carbocycles and heterocycles is a fundamental task for an organic chemist.

Starting from the Nazarov reaction, the replacement of the double-bond by a cyclopropane could give access to larger ring systems via a 'homo-Nazarov' cyclization
(Scheme 1). ${ }^{[2]}$ Different from the concerted Nazarov cyclization, the cyclization of vinyl-cyclopropyl ketones proceeds via a stepwise process involving a carbocationic intermediate III.

In 2009, we reported the development of the first catalytic method for the homoNazarov reaction of vinyl-cyclopropylketones using a catalytic amount of a Brønsted acid in acetonitrile. ${ }^{[3]}$ Although the discovery of the new catalytic conditions for the formal homo-Nazarov cyclization allowed the application of our method to several unprecedented heterocyclic structures, the required presence of an aromatic group to stabilize the carbocationic intermediate still constituted a severe limitation in terms of scope.

We report herein the extension of the methodology obtained replacing the aromatic stabilizing group by a heteroatom
(Scheme 2). Indeed, the presence of a heteroatom would assure a good stabilization of the formed carbocation, as demonstrated by the broad literature of donor-acceptor cyclopropanes, ${ }^{[4]}$ and would extend the range of structures accessible. In particular, the cyclohexylamines which could be obtained through the cyclization of aminocyclopropanes can be found in several natural alkaloids such as aspidospermidine (1), aspidofractinine (2), strychnine (3), vindoline (4), vindolinine (5), vinblastine (6) and vincristine (7) (Scheme 2). An aminocyclopropane could be used as precursor of an iminium intermediate in the formal homo-Nazarov cyclization. However, aminocyclopropanes with no electronwithdrawing groups are unstable and the presence of an electron-withdrawing group on the nitrogen would be required to prevent the opening of the cyclopropane ring.
${ }^{*}$ Correspondence: Prof. Dr. J. Waser

Ecole Polytechnique Fédérale de Lausanne

Laboratory of Catalysis and Organic Synthesis

EPFL SB ISIC LCSO

BCH 4306

$\mathrm{CH}-1015$ Lausanne

Tel.: +41216939388

Fax: +41216939700

E-mail: jerome.waser@epfl.ch

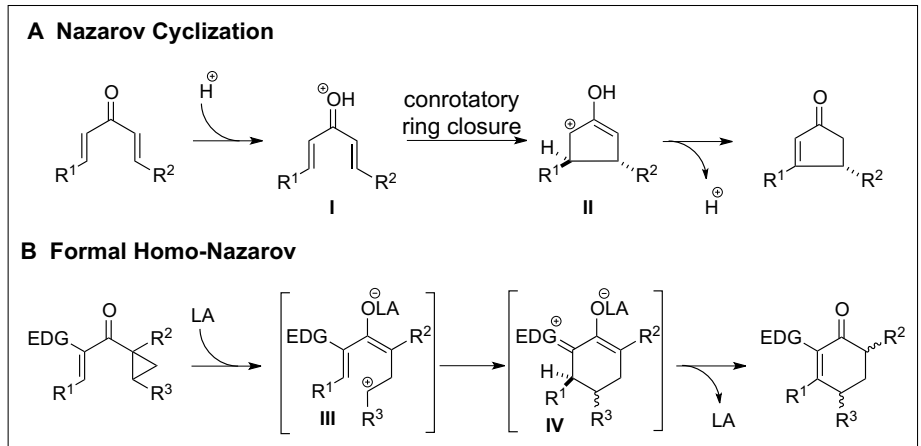

Scheme 1. Mechanism of the Nazarov and formal homo-Nazarov reaction. 


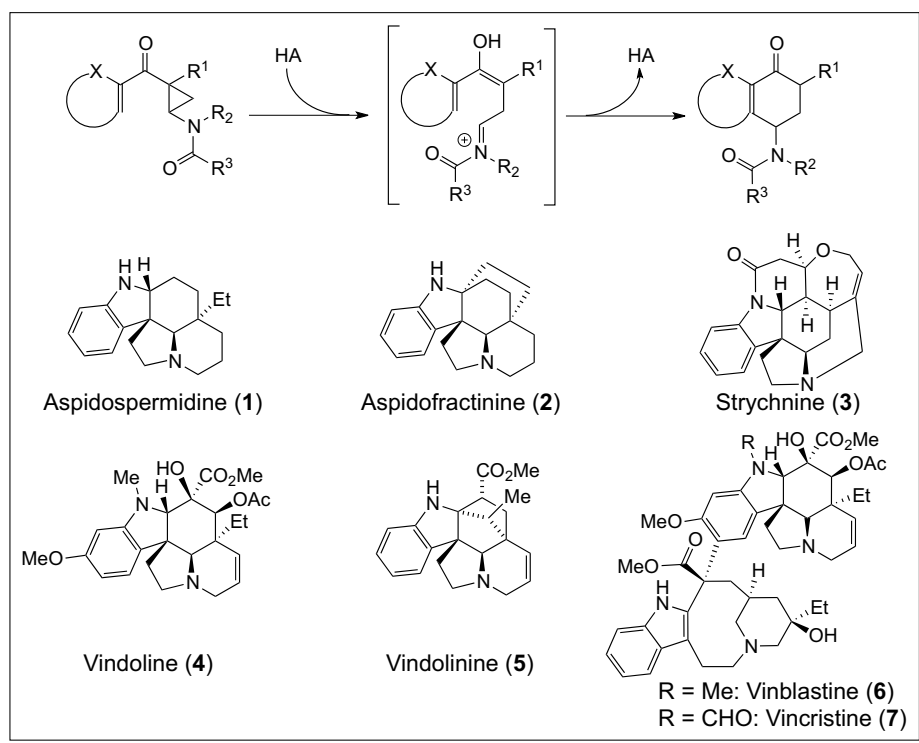

Scheme 2. Formal homo-Nazarov cyclization of aminocyclopropanes and alkaloid natural products containing a cyclohexylamine ring

In this context, acylamino cyclopropanes represent a good compromise in terms of reactivity, since they are sufficiently electron-rich to promote the ring opening and stabilize the formed cation as an acyliminium species (I), but are stable enough to be isolated (Scheme 2).

We propose herein the first utilization of the catalytic formal homo-Nazarov cyclization of aminocyclopropanes in the synthesis of a natural product. Based on this method, a formal total synthesis of the Aspidosperma alkaloid aspidospermidine (1) is presented.

Moreover, the versatility of our cyclization method is demonstrated by its successful application in the total synthesis of the natural alkaloid goniomitine (21), accomplished in 13 linear steps with an overall yield of $11 \%$.

Finally, the scope and limitations of our methodology are investigated for a wide range of vinyl-cyclopropyl ketones.

\section{Formal Total Synthesis of Aspidospermidine}

When considering potential targets for the formal homo-Nazarov cyclization of aminocyclopropanes, we decided to attempt first a synthesis of the Aspidosperma alkaloid aspidospermidine (1). We selected this alkaloid, isolated from the bark of Aspidosperma quebracho blanco and other species native of South America, ${ }^{[5]}$ since it is the simplest natural product containing the tetracyclic scaffold also present in many more complex alkaloids. A new convergent approach to the total synthesis of Aspidosperma alkaloids would give access to a wide range of potentially bioactive natural compounds and synthetic analogues.

Starting from commercially available $\delta$-valerolactam $(\mathbf{8})$, a sequence of protection, alkylation, reduction and dehydration afforded enamide 9 in good yield (Scheme 3). ${ }^{[6]}$ The benzyl-carbamoyl protecting group was chosen due to its tolerance towards acidic conditions and its easy removal under mild conditions (for example via hydrogenation), as well as in order to obtain the desired electron-density on the nitrogen.

It was important to finely tune the properties of the catalyst and the reaction time in order to obtain reproducible yield for the cyclopropanation of 9 . On preparative scale, a diasteromeric mixture $(1: 1 \mathrm{dr})$ of cyclopropane ethyl esters 10a,b was obtained using copper (I) triflate and ethyl diazoacetate in a reproducible $76 \%$ of yield. Endo ester 10b was then isomerized into the more stable exo form 10a with catalytic $\mathrm{BF}_{3} \cdot \mathrm{OEt}_{2}$. This isomerization, first introduced by Grieco, was proposed to occur via a ketene iminium salt. ${ }^{[6]}$ Ester 10a was then hydrolyzed to give a single diastereoisomers of carboxylic acid 11. The choice of the right conditions to convert the sensitive cyclopropane $\mathbf{1 1}$ into the Weinreb amide $\mathbf{1 2}$ in good yield was crucial. After a short screening of coupling reagents, we selected 4-(4,6-dimethoxy-1,3,5-triazin2-yl)-4-methylmorpholinium chloride (DMTMM) since it allowed the formation of the amide $\mathbf{1 2}$ in good yield and without further purification. With these encouraging results in hand, we decided to synthesize the free indole cyclization precursor 14. The use of indole $N$-carboxylates pioneered by Katritzki ${ }^{[7]}$ was considered the more appropriate choice. Indeed, the carboxy group presented the advantage to direct selectively the lithiation on the $\mathrm{C}(2)$ position of indole and to protect the indole nitrogen at the same time. Furthermore, the obtained carboxy indole is labile and underwent decarboxylation upon work up to give directly the unprotected product. Nevertheless, the literature about this method included only examples with more simple substrates. Using isolated and recrystallized indole- $N$-carboxylic acid $\mathbf{1 3}$ we were pleased to isolate the precursor 14 for the formal homo Nazarov cyclization in good yield.

Surprisingly, when we attempted the cyclization of $\mathbf{1 4}$ using our standard conditions for the formal homo-Nazarov reaction, two different compounds were
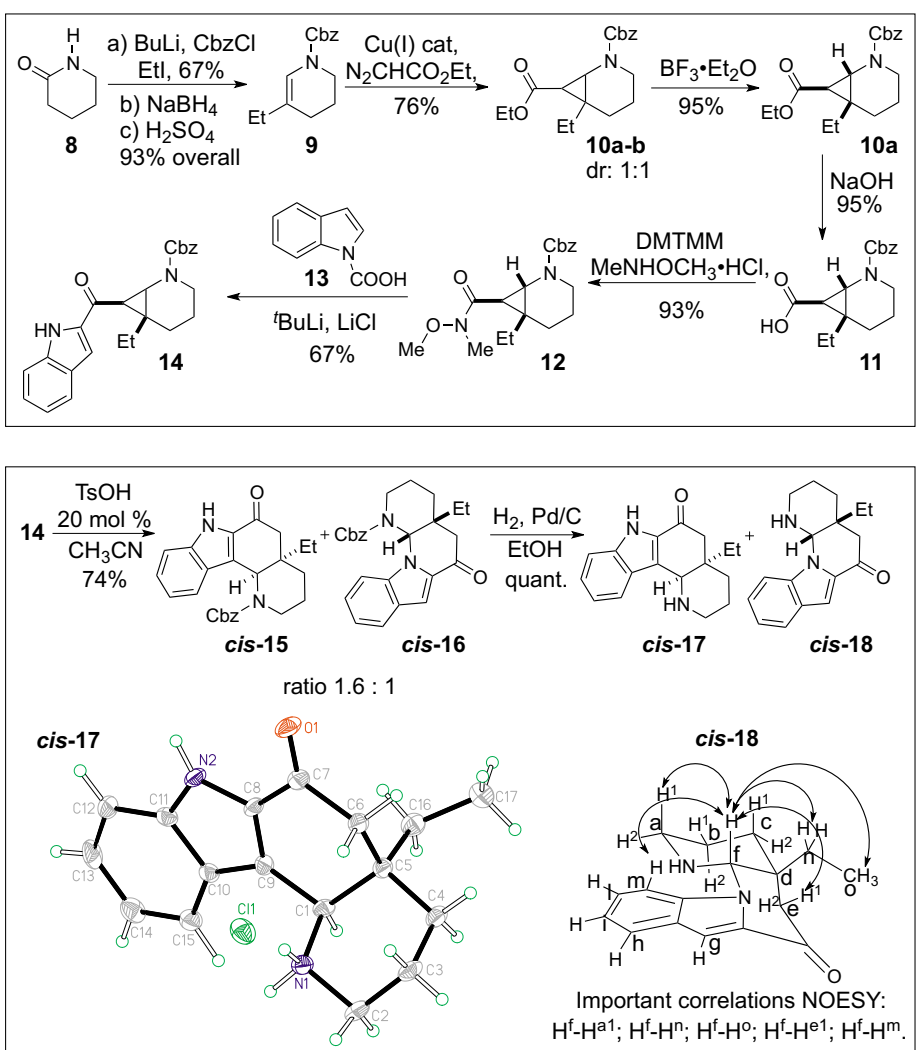

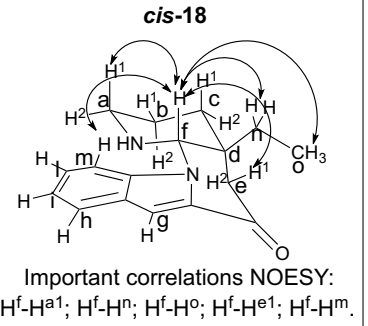

Scheme 3. Synthesis of indole-substituted cyclopropane 14.

Scheme 4. Cyclization of cyclopropane 14 and determination of the relative configuration of cis-17 and cis-18. 
isolated. NMR and X-ray analysis carried out after separation of the two cyclization products and removal of the Cbz protecting group allowed the identification of the desired product cis-15 derived from the attack on indole at the $\mathrm{C}(3)$ position and the compound cis-16 resulting from cyclization on the $\mathrm{N}(1)$ position in a ratio of 1.6:1 (Scheme 4). The isolation of free amine cis-17 accomplished the successful formal total synthesis of aspidospermidine (1), since this intermediate had been reported in the synthesis by Wenkert and Hudlicky. ${ }^{[8]}$

\section{Selective Cyclization}

In order to improve the selectivity towards $\mathrm{C}-\mathrm{C}$ cyclization, we tested several combinations of acid catalysts and solvents (Scheme 5). We were pleased to note that softer Lewis acid such as copper salts increased dramatically the regioselectivity toward the $\mathrm{C}-\mathrm{C}$ cyclization allowing the formation of the desired cyclic compound cis-15 as a pure diastereoisomer in good yield. The N-cyclization product cis-16 was also highly interesting, since it represented the tetracyclic core of goniomitine (21). Considering the rarity of this scaffold in nature, the few examples of reported synthetic approaches ${ }^{[9]}$ and the absence of any information on its bioactivity, we decided to optimize the cyclization towards the formation of the $\mathrm{N}(1)$ cyclization product. During the screening of several different solvents we were delighted to find a remarkable switch of selectivity when $p$-toluenesulfonic acid was tested in dichloromethane. The formation of the goniomitine scaffold cis-16 was obtained with high yield and diastereoselectivity.

One of the possible explanations for the observed selectivity could lie in the hard/soft properties of the generated enol intermediates which allowed the selective formation of either the kinetic or the thermodynamic product. When the aminocyclopropane is activated with $\mathrm{Cu}(\mathrm{OTf})_{2}$ the formed copper-bound enolate would have a 'soft' character, which could favor the attack of the acyl iminium on the softer $\mathrm{C}(3)$ indole position. Proton-transfer and rearomatization of the cyclization product lead to the more stable thermodynamic product cis-15. Moreover, the polar solvent is fundamental to stabilize the charged iminium intermediate so that the charge-controlled reaction with the nitrogen becomes slower compared with the attack from the carbon atom.

On the other hand, the use of $\mathrm{TsOH}$ would generate a hard enol which could lead to the attack of the iminium on the harder N(1) position of indole. The noncoordinating effect of methylene chloride, would hence favor a fast charge-controlled attack on the harder nitrogen. The formed intermediate, through deprotonation and tautomerization would yield the kinetic product $c i s-16$

In order to confirm this hypothesis, we analyzed the interconversion of the two cyclic compounds under the reaction conditions used to obtain the opposite regioisomer. In presence of copper triflate in acetonitrile, it was possible to convert the cyclic compound cis-16 into the regioisomer cis-15, while no interconversion was observed when cis-15 was submitted to $p$ toluenesulfonic acid in dichloromethane.
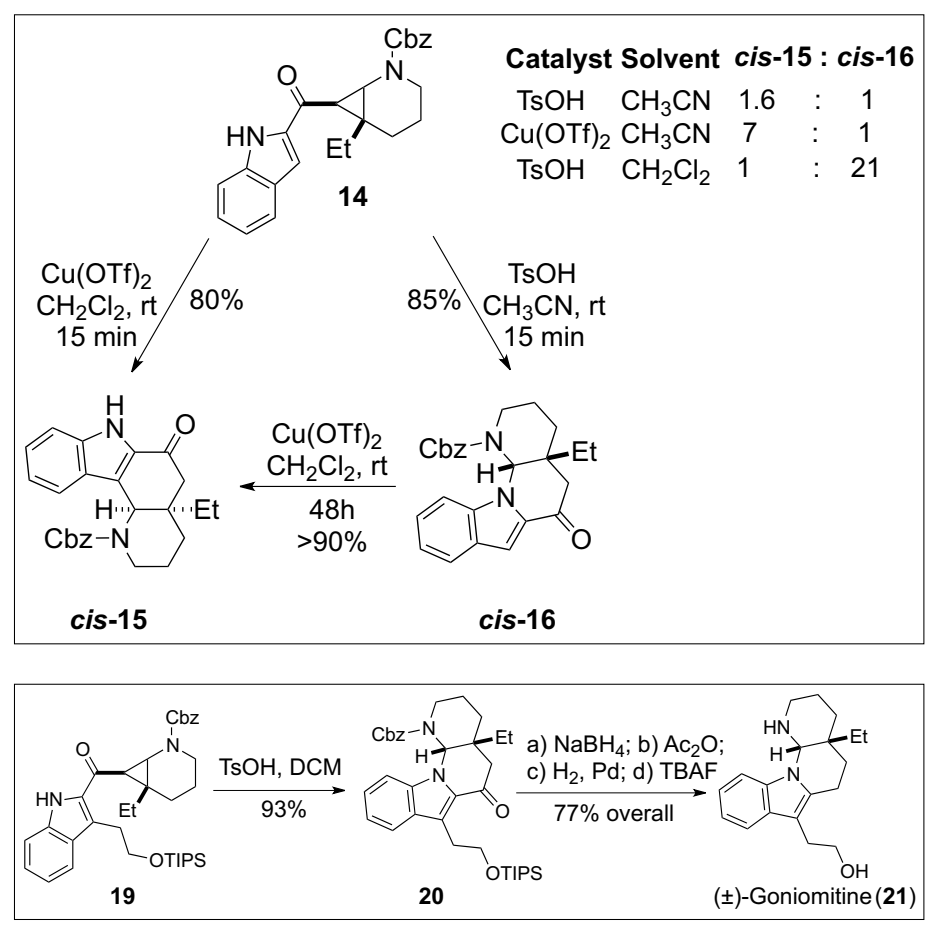

Scheme 5. Regioand diastereoselective cyclization of aminocyclopropane 14.

Scheme 6. Total synthesis of goniomitine (21).

Moreover, in both the cyclization mechanism, the regioisomers were obtained as pure diastereoisomers. Qualitative analysis of the possible transition states suggested that a classical Fürst-Plattner stereocontrol could be the basis for the high stereoselectivity observed for this particular cyclization. ${ }^{[10]}$

\section{Total Synthesis of Goniomitine}

In order to finish the total synthesis of goniomitine, we considered a more convergent approach starting from the cyclization precursor 19. Cyclopropane 19 was obtained from the addition of a bislithiated salt of TIPS-protected and $N$-carboxylated tryptophol on Weinreb amide $\mathbf{1 2}$.

The indole 19 was then cyclized in presence of catalytic $p$-toluenesulfonic acid in dichloromethane (Scheme 6). The presence of the alkyl chain at $\mathrm{C}(3)$ had no negative influence on the cyclization rate, and the desired product $\mathbf{2 0}$ was obtained in good yield as a pure diastereoisomer.

The carbonyl group was then reduced with sodium borohydride and the resulting alcohol acetylated in order to facilitate the cleavage of the $\mathrm{C}-\mathrm{O}$ bond. The acetate and the benzyl carbamate were cleaved during hydrogenolysis and the deprotection of the primary alcohol using TBAF finally gave goniomitine (21) in $77 \%$ overall yield from 20.

The total synthesis of goniomitine (21) was accomplished in a linear sequence of 13 steps with an overall yield of $11 \%$. ${ }^{111]}$ This synthesis represents up to date the most efficient way to obtain goniomitine (21) as a racemic mixture. Moreover our convergent approach could be applied to introduce a broad structural diversity on the indole and piperidine moiety.

\section{Scope Extension}

With the optimized conditions for the regioselective cyclization in hand, we decided to synthesize a further series of substrates in order to extend the scope of this new reaction (Scheme 7). Modifications of the hetero-aromatic part of the molecule were first considered. Using the conditions optimized for the cyclization of aminocyclopropane 14, we were pleased to observe a high yielding cyclization with a complete control on the regiochemistry for methoxyindoles 22a and 22b. When pyran was used as nucleophile (23), the cyclization product 29 was obtained using $p$-toluenesulfonic acid as catalyst in acetonitrile.

The scope was then extended to acyclic aminocyclopropanes (24 and $\mathbf{2 5}$ ) and an oxycyclopropane (26). ${ }^{[12]}$ For N-methyl indole 24, cyclization was successful, but a 


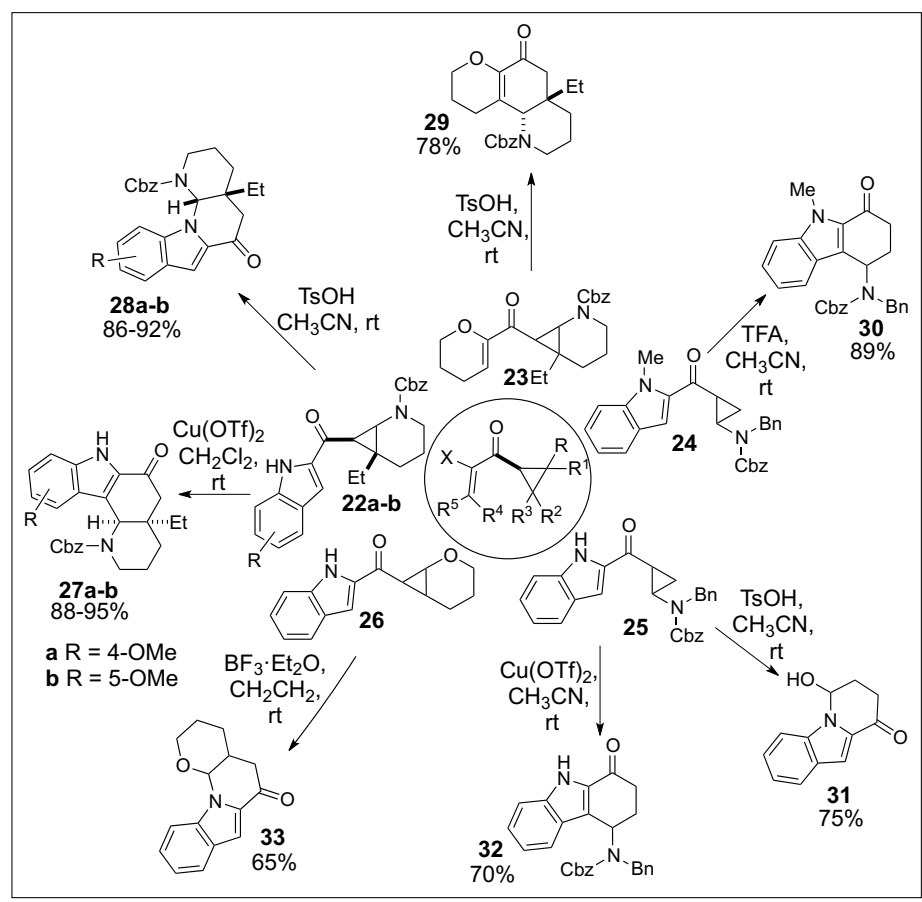

Scheme 7. Extension of the cyclization scope.

milder acid (TFA) had to be used to prevent formation of side products via hydrolysis and elimination to give a carbazole. In the case of unprotected indole $\mathbf{2 5}$, cyclization on either $\mathrm{C}(3)$ or $\mathrm{N}(1)$ could be again obtained in dependence on the choice of catalyst. Oxycyclopropane 26 gave N(1) cyclization product 33 exclusively, but required the use of a stronger Lewis acid.

\section{Conclusion}

In conclusion, we have developed the first catalytic formal homo-Nazarov reaction and discovered also other types of cyclizations for donor-acceptor activated cyclopropanes. The versatility of our methodology has been showcased through sev- eral successful applications in the synthesis of complex polycyclic scaffolds and the total synthesis of natural products.

In a broader sense, our method allowed access to iminiums under mild conditions based on the release of ring strain and cross polarization. When considering the utility of such reactive intermediate in the synthesis of complex structure, the formal homo-Nazarov cyclization is expected to be broadly applicable for the synthesis of other natural and synthetic bioactive compounds. Moreover, the development of an asymmetric cyclopropanation method would allow an asymmetric entry into important building blocks for the synthesis of natural products.

The principle of cyclopropane activation discovered in this project are certainly not limited to the formal homo-Nazarov process, and further applications in other type of cyclization or intermolecular annulation reactions can be expected in the future. ${ }^{[13]}$

Received: January 13, 2012

[1] J. Clardy, C. Walsh, Nature 2004, 432, 829.

[2] a) W. S. Murphy, S. Wattanasin, Tetrahedron Lett. 1980, 21, 1887; b) O. Tsuge, S. Kanemasa, T. Otsuka, T. Suzuki, Bull. Chem. Soc. Jpn. 1988, 61, 2897; c) F. De Simone, J. Waser, Chimia 2009, 63, 162.

[3] F. De Simone, J. Andres, R. Torosantucci, J. Waser, Org. Lett. 2009, 11, 1023.

[4] a) H. U. Reissig, R. Zimmer, Chem. Rev. 2003, 103, 1151; b) F. Gnad, O. Reiser, Chem. Rev. 2003, 103, 1603; c) M. Yu, B. L. Pagenkopf, Tetrahedron 2005, 61, 321; d) C. A. Carson, M. A. Kerr, Chem. Soc. Rev. 2009, 38, 3051; e) F. De Simone, J. Waser, Synthesis 2009, 3353; f) T. P. Lebold, M. A. Kerr,. Pure Appl. Chem. 2010, 82, 1797.

[5] G. Fraude, Berichte 1878, 11, 2189.

[6] P. A. Grieco, M. D. Kaufman, J. Org. Chem. 1999, 64, 7586.

[7] A. R. Katritzky, K. Akutagawa, Tetrahedron Lett. 1985, 26, 5935.

[8] E. Wenkert, T. Hudlicky, J. Org. Chem. 1988, 53, 1953.

[9] a) C. Hashimoto, H. P. Husson, Tetrahedron Lett. 1988, 29, 4563; b) S. Takano, T. Sato, K. Inomata, K. Ogasawara, J. Chem. Soc. Chem. Commun. 1991, 462; c) G. Lewin, C. Schaeffer, Nat. Prod. Lett. 1995, 7, 227; d) G. Lewin, C. Schaeffer, P. H. Lambert, J. Org. Chem. 1995, 60, 3282; e) G. Lewin, C. Schaeffer, R. Hocquemiller, E. Jacoby, S. Leonce, A. Pierre, G. Atassi, Heterocycles 2000, 53, 2353; f) C. L. Morales, B. L. Pagenkopf, Org. Lett. 2008, 10, 157; g) M. Mizutani, F. Inagaki, T. Nakanishi, C. Yanagihara, I. Tamai, C. Mukai, Org. Lett. 2011, 13, 1796.

[10] F. De Simone, J. Gertsch, J. Waser, Synlett 2011, 589.

[11] F. De Simone, J. Gertsch, J. Waser, Angew. Chem. Int. Ed. 2010, 49, 5767.

[12] F. De Simone, T. Saget, F. Benfatti, S. Almeida, J. Waser, Chem. Eur J. 2011, 17, 14527.

[13] a) F. De Nanteuil, J. Waser, Angew. Chem., Int. Ed. 2011, 50, 12075. b) F. Benfatti, F. De Nanteuil, J. Waser, Org. Lett. 2012, 14, 386. 